\title{
A Study of a Single Robot Interacting with Groups of Children in a Rotation Game Scenario ${ }^{1}$
}

\author{
René te Boekhorst, Michael L. Walters, Kheng Lee Koay, Kerstin Dautenhahn, Chrystopher L. Nehaniv \\ \{R.teBoekhorst, M.L.Walters, K.L.Koay, K.Dautenhahn, C.L.Nehaniv\} @herts.ac.uk \\ Adaptive Systems Research Group \\ School of Computer Science \\ University of Hertfordshire \\ College Lane, Hatfield, Herts. AL10 9AB, United Kingdom
}

\begin{abstract}
We tested the hypothesis that children are more attentive to a robot if the robot appears to be interested in the children. In addition, we investigated if and how the quality and quantity of a child's attentive behaviour varies with the distance to the robot, reflecting the notion of "social spaces". Hereto, 16 groups of up to 10 children each were engaged in a play scenario in which they had to move closer to a robot over 6 successive rounds. The robot was endowed with a "camera eye" and an arm and hand. The camera could either be nonmoving ("static") or actively "searching" ("active searching"), giving the impression it was trying to select a child to focus on. Likewise, the arm and hand could either be fixed in a permanent pointing position ("permanent pointing") or actively rise to point selectively at a particular child when it stopped facing it ("selective pointing"). The results showed that:

1) The mean frequency of overall attentive behaviour by the children (including attention towards other children) was significantly higher when the robot was not selectively pointing at the children and independent of the state of the camera.

2) "Looking at" was the most frequently scored attentive activity for the children and was mainly targeted to the robot, but not correlated with any of their other attentive activities.

3) There was an interaction effect between the state of the camera and of the pointer: looking at the robot by the children occurred significantly more often when the camera and the arm were consistent in signalling apparent interest (i.e. camera "active searching" and hand "selectively pointing" or camera "static" and hand "permanently pointing").

4) There was no demonstrable effect of distance to the robot on the overall attentive behaviour of the children.
\end{abstract}

Index Terms - Human-Robot Interaction, Social Spaces, Social Interaction, Social Distances, Social Robot, Deixis.

\section{INTRODUCTION}

The impact of design features on the attraction and acceptance of agents by humans is a major topic in HumanAgent Interaction research [Breazeal 2002, Dautenhahn et al. 2002, Dryer 1999]. Acceptance hinges on feelings of control and comfort [Norman 1994]. Whereas control is about having information (knowing how an agent can be operated) to enable manipulative and corrective operations, comfort has to do with the social aspects of acceptability, such as trust. Trust in the agent, in turn, is related to the reassurance that all is working to plan and therefore requires the actions of the agent to be understandable (knowing what an agent does) [Norman, 1997]. Critical for the latter is that an agent makes its presence and actions known to the human. For this, attracting (and keeping) attention by the agent is of principal importance and the role of personification is frequently debated in this context (see for example Erickson [1997]; Takeuchi et al. [1995]). A number of researchers assume that a believable personification is mainly obtained by an anthropomorphic shape. However, as will be argued in this paper, a believable and consistent combination of actions might be just as important (cf. [Dautenhahn 1998, Dautenhahn and Nehaniv 2000]).

The above issues have originally been raised for the design of software agents [Bradshaw, 1997], but they also directly apply to the design of hardware agents (robots). Apart from the role of attention in building up trust, a special topic that needs to be considered in the case of robots (but that does not apply to virtual agents) is the feeling of physical safety, which in turn is directly related to the distance between human and robot.

The study of attraction towards a robot on the one hand and feelings of comfort on the other hand (reflected by the association between attentive behaviour and distance to the robot) is of obvious importance to the emergent field of assistive robotics in general and to the design of robots for use in the home in particular (for an overview of socially interactive robots, see Fong et al. [2003]). As such, the investigation of this research question is part of our contribution to the COGNIRON project [COGNIRON 2004], of which the major objective is to develop a robot that serves humans as a companion in their daily lives.

To formulate the research question explicitly in the wider area of Human-Robot Interaction, we stress the notion of interaction by considering attention in the context of embodied interaction. Eye-gaze direction and deictic gestures such as pointing with the hand play important early roles in the ontogeny of inter-subjectivity and shared attention as well as linguistic and cognitive abilities in the development of human children; and they continue to play a crucial role in non-verbal communication in adults, depending crucially on embodied interaction [see, e.g. Kita 2003].

\footnotetext{
${ }^{1}$ The work described in this paper was conducted within the EU Integrated Project COGNIRON ("The Cognitive Robot Companion") and was funded by the European Commission Division FP6-IST Future and Emerging Technologies under Contract FP6-002020.
} 
These considerations have lead to the following working hypothesis: humans are more attentive towards the robot if the robot appears to be "interested" in the people present. ${ }^{2}$ We implemented this in two ways: First, the camera on top of the robot was engineered so that it could either remain immobile ("static") or move around its axis and thus give the impression of being a searching "eye". Second, the robot was endowed with an arm and a human shaped "hand" that could rise to point to a particular, chosen person within a group of subjects ("selective pointing" as in contrast to the default state of "permanent pointing" in which the hand always points forward).
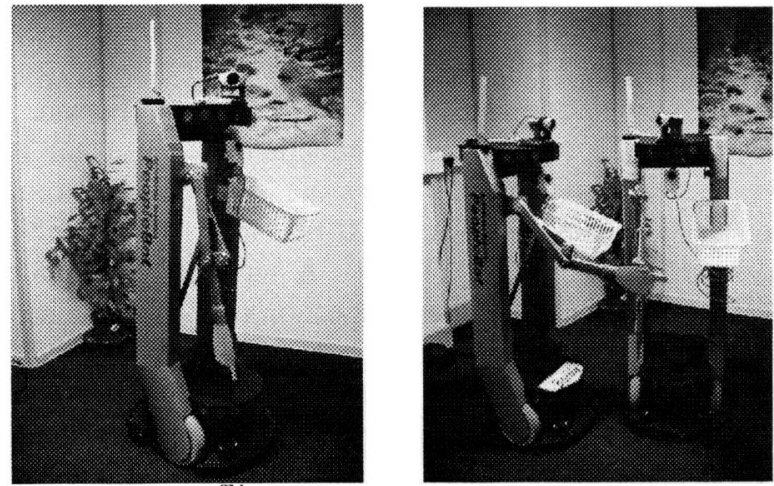

Fig. 1: The PeopleBot ${ }^{\mathrm{TM}}$ robots fitted with a pointer (in raised and lowered positions) and basket.

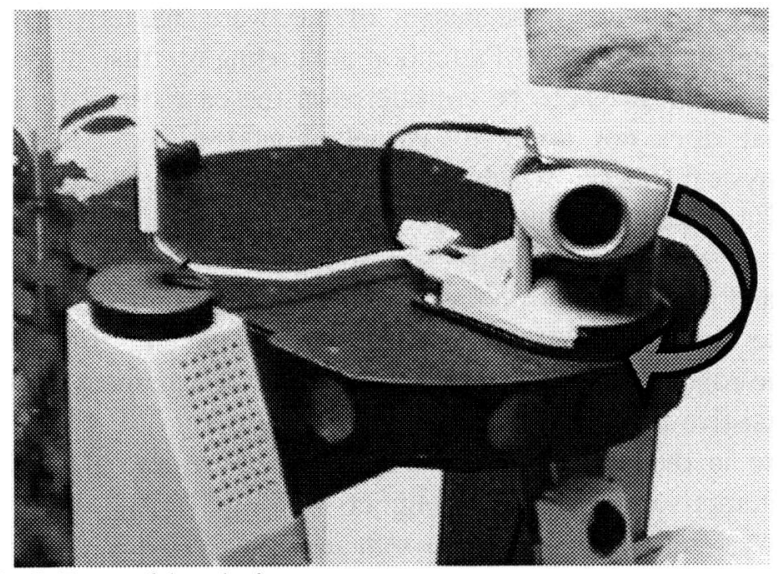

Fig. 2: Extent of the robot's camera movement

Experiments were carried out in which groups of up to 10 school children were confronted with such a robot in a play scenario. The behaviour of the children was scored at various distances to the robot and compared under all the possible combinations of the state of camera ("static" or "active searching") and pointer ("permanent pointing" or "selective pointing").

\section{METHODS}

The study took place in June 2004, taking advantage of a larger event run by the FP5 European Project VICTEC [2003]. For the VICTEC project, about 400 school children (all but two born and raised in the U.K., aged between 9 and

\footnotetext{
${ }^{2}$ We are not claiming that our robot was genuinely interested in the children, but refer to the usage of cues that give the appearance of "interest" from an observer perspective.
}

11 year and chosen from 10 schools in the Hertfordshire area) visited the university and 194 of these participated in the experimental sessions described in this paper. These sessions were used to run interactive games with groups of children and a single robot. The main aims of this study were to investigate interaction styles in a group scenario involving children and how they depend on proximity to the robot. In particular, we wanted to find out how a robot can attract the attention (by using combinations of two attention attraction devices) of humans and how humans react to being the focus of the robot's attention. The study was conducted using commercially available, human-scaled, PeopleBot robots [ActivMedia, 2005].

\section{A. Experimental Set-up}

The sessions took a maximum time of 30 minutes and were run in parallel in two separate rooms. Six sessions were carried out each day for four days. In each session a robot and a group of children were involved in an interactive "rotation game" consisting of six rounds. The two rooms to be used for the study were both $10 \mathrm{~m}$ long by $6 \mathrm{~m}$ wide. Each room contained a PeopleBot robot in the centre, initially covered by a black plastic sack. The room was marked out into 6 concentric zones around the robot at $0.5 \mathrm{~m}$ radii. There was video recording equipment set up in each room consisting of two fixed video cameras, and a feed from the robot onboard video camera. The videos were time coded, for later synchronization and evaluation, and each day's sessions were recorded on a new set of tapes. The tapes were then downloaded and stored on computer readable compressed format (mpeg 1 or 2) on CD or DVD disk, for later evaluation. The robots were controlled in a semiautonomous manner, with the operators retaining control of starting the games, and also making sure that the robots did not point to an empty space when selecting (by stopping the turning robot when it was facing) a child. In the "permanent pointing" mode, the pointer did not move, but was constantly extended. In "selective pointing" mode, the pointer was lowered most of the time, but raised when a child was selected. The two robot operators were hidden in an adjoining third room along with the wireless network, recording equipment, and the various data processing computers. The game (but not the purpose of the experiment) was explained to the children by an experimenter who initiated the game with a signal to the robot (seen by the robot operators). During the experiment, behaviour of the children was recorded by the video camera and another observer took notes of any particularly interesting interactions.

During the game, the robot revolved in the middle of a circle of children with various slow, fast, reverse and "teasing" movements to keep the children interested. After 30 seconds the robot stopped when it faced the nearest child and "selected" it by beeping twice, stopping in front of that child and - depending on the experimental condition raising the pointer toward the child.

The chosen child was allowed to pick up a small present from a basket carried by the robot and then left the game The remaining children moved $0.5 \mathrm{~m}$ closer (to the next zone) to the robot and the game was repeated for a total of six 
rounds. At the end of the game, all the children that were left over received an identical present.

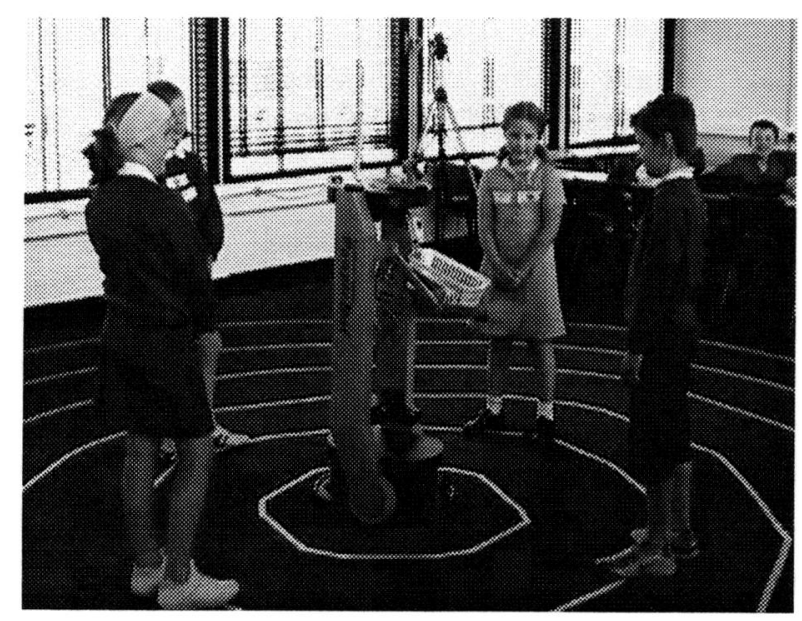

Fig. 3: A child being selected by the robot while playing the Rotation Game with pointer rising to point to him (selective pointer mode). Spatial zones are marked on floor.

The permanent pointer was always in the raised position pointing forward. The selective pointer was normally in the lowered position (pointing to the floor) but when selecting a child the pointer was raised so that the hand was pointing with the finger at the person or object directly in front of the robot (Figs. 1 and 3) and then lowered once more before the child took the present.

In addition to a selective pointing or a permanent pointing hand, the other variable condition was the state of the camera. The camera was either stationary ("static") or panning from side to side in a random manner ("active searching") (Fig. 2). Hence, the overall setup for statistical analysis is a $2 \times 2$ design of four experimental conditions (fig. 4):

1. Camera static and permanent pointing

2. Camera static and selective pointing

3. Camera active searching and permanent pointing

4. Camera active searching and selective pointing

The dependent variables are the frequencies of activities scored from a focal child (see next section). Out of twentyfour rotation games, sixteen giving the best quality video data were chosen for analysis. This resulted in a final data set consisting of four different groups for each of the four experimental conditions.

\section{B. Collection, Processing and Analysis of the Data}

From each videoed session, one child was selected for analysis, thus guaranteeing independence of data. Selection was based on the visibility of the child in the video clip and the duration of time he or she spent playing the game (i.e. children that dropped out of the game after the first two rounds were not considered). After preliminary viewing of the videos, (not necessarily robot-directed) "Pointing at", "Talking to" and "Looking at" were selected as behavioural activities for an initial analysis. These activities were understood as signs of interest and attention shown by the children to the environment (including the robot and other persons). Their summed frequency is considered as overall "attentive behaviour".

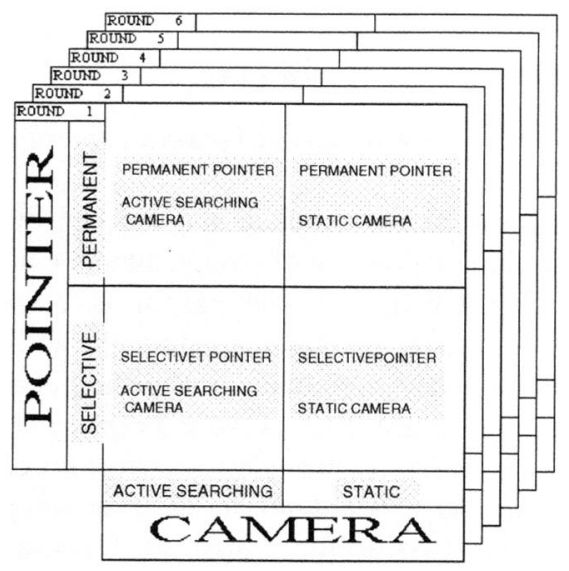

Fig. 4: The four experimental conditions, showing four combinations of active searching/static camera and permanent/selective pointer used for playing the rotation game with randomly selected groups of children. (Each lasted for six rounds, i.e. six children from each typical group of ten children were selected by the robot in the course of a game)

The selected activities were coded using the program ANNOTATOR developed by the University of Hertfordshire Adaptive Systems Research Group. AnNOTATor enables the interactive recording of behavioural variables at fixed time intervals: coded activities can be scored and saved in a worksheet during the simultaneous display of a video clip of an experiment. This methodology of behavioural analysis has previously proved beneficial in analysing human-robot interaction, e.g. [Dautenhahn and Werry, 2002], [Robins et al. 2004]. A one-second time unit was adopted for our purposes and attentive behavioural activities were scored according to the target they were directed at (Robot, Other Children, and Other Persons - such as teacher or the instructing researcher - and "Unknown Target"). For the purpose of the analysis, attention targets coded as Other Person and Unknown Target were joined together into a lump-category "Else". The final data set thus consists of the frequency of the "Talking to", "Pointing at" and "Looking at" three targets (Robot, Other Children and Else) displayed by one child selected from each of four groups, giving a total of 16 data points.

The coding started from the beginning of the game to either the end of the game (when all remaining children were asked to take a present from the robot), or the end of the game for the child selected for coding (when he or she was picked by the robot). During the rotation game, the moment when children moved to the next circular zone was also coded (from the moment the child selected for coding had both feet in the next circle). Zones were coded from 1 (inner-most circle) to 5 (furthest from the robot).

We found a strong effect of the duration of the game on the frequency by which a subject displayed attentive behaviour. As a result of this, statistical tests could only be performed after controlling for the effect of duration or by making (matched) comparisons within subjects.

In the next section, we will respectively address: i) differences in overall attentive behaviour between the 
experimental conditions; ii) the allocation of directed attention; iii) the association between directed attention and the experimental conditions, and iv) the effect of proximity to the robot on overall attentive behaviour.

\section{RESULTS}

\section{A. Differences in overall activity between experimental conditions.}

The effect of time was taken into account by using the residuals from the regression of overall attentive activity (the sum of looking, talking and pointing) on the duration of an experiment. Residuals were computed for the activity scores of each subject and were found to meet the assumptions of analysis of variance (Bartlett's, Cox and Hartley statistics for homogeneity of variance; normality of error; no correlations between means and standard deviations of the samples).

A repeated two factor (State of Camera = active searching, static, and State of the Pointer = selective, permanent) ANOVA was carried out. A significant effect for the state of the pointer was found $\left(\mathrm{F}_{1,12}=5.73, \mathrm{p}=\right.$ $0.034)$ and indicated that subjects exhibited more attentive behaviour (including towards each other) when the pointer was permanent as compared to the selective pointer (Fig. 4). Some of the directed attention variables (after timecorrection, i.e. the residuals from the regression on duration) appeared to be significantly correlated. For example pointing to the robot was significantly correlated with talking to the other children (Pearson correlation, $r=0.59$ ).

These correlations may be partly due to simultaneous attentive activity: when pointing to the robot, subjects may have talked about the robot at the same time with their group members. However, the significant correlation between talking to the robot and pointing at other children $(r=0.57)$ is less easy to explain as is the absence of correlations between talking to and pointing at the robot.

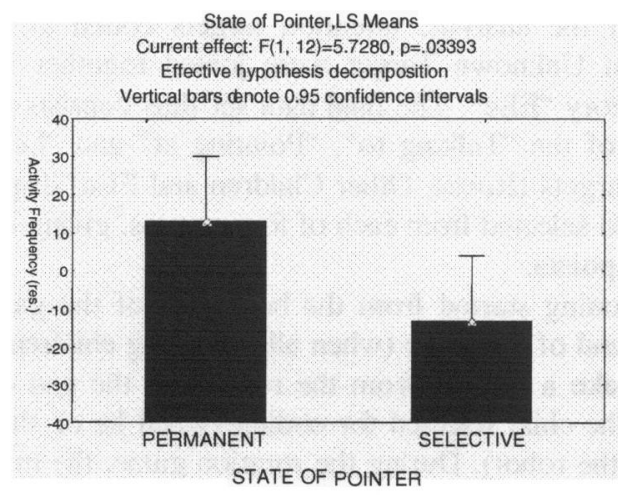

Fig. 4: The effect of the mode of the robot's pointing on the overall attentive behaviour of subjects (including attentiveness towards each other).

\section{B. Allocation of Directed Attention}

No significant effects were revealed for the state of the camera or for the interaction between the states of camera and pointer. As an alternative procedure an Analysis of Covariance was performed on the raw scores, but with Duration as co-variable. The results were identical to those reported above.
In the previous tests, only the overall attentive behaviour was considered. Table 1 shows how attentive behaviour was allocated by the subjects. "Looking at" was the most frequently scored activity and was mainly directed towards the robot. "Pointing at" and "Talking to" only made up for a small proportion of the overall activities and were in the majority of cases targeted towards the other children.

TABLE 1.

DISTRIBUTION OF THE DIRECTED ATTENTION VARIABLES

\begin{tabular}{|c|c|c|c|c|}
\hline \multirow[b]{2}{*}{ ATTENTION VARIABLE } & \multicolumn{4}{|c|}{ DIRECTION } \\
\hline & at robot & children & else & Totals: \\
\hline Looking & 3066 & 1281 & 612 & 4959 \\
\hline Talking & 59 & 164 & 5 & 228 \\
\hline Pointing & 9 & 22 & 2 & 33 \\
\hline Totals: & 3134 & 1467 & 619 & 5220 \\
\hline
\end{tabular}

The correlation between talking to the robot and pointing at their classmates could be due to attempts to influence the robot. E.g. a child would say "pick him!" to the robot, while pointing to their classmates. Furthermore, it is noteworthy that although "Looking at" was the dominant activity it appeared not to be correlated with any of the other directed attention variables. Further investigations need to be carried out to clarify these matters.

\section{Associations between Directed Attention and Experimental Conditions.}

In order to assess the children's attention towards the robot in a single measure, for each attention variable we calculated the "AttentionBy•X" variable (where $\mathrm{X}$ is one of the three attention variables "Looking at", "Talking to" or "Pointing at"):

AttentionBy $\bullet$ AttentionVariable $=\frac{\text { AttentionVariable }- \text { Attention Variable } \bullet \text { notrobot }}{\text { AttentionVariable }} \times 100$

In this formula notrobot refers to the frequency of that attention variable directed at children plus "else" (i.e. not targeted to the robot). Similarly, •total is the total frequency of the considered attention variable. For example, attention towards the robot by "Looking" at the robot is formulated as:

Attention By $\bullet$ Looking $=\frac{\text { Looking }- \text { Looking } \bullet \text { notrobot }}{\text { Looking } \cdot \text { Total }} \times 100$

The division by the total frequency makes this measure independent of duration.

Pointer ${ }^{*}$ Camera; LS Means

Current effect: $F(1,12)=7.8053, p=.01623$

Vertical bars denote 0.95 confidence intervals

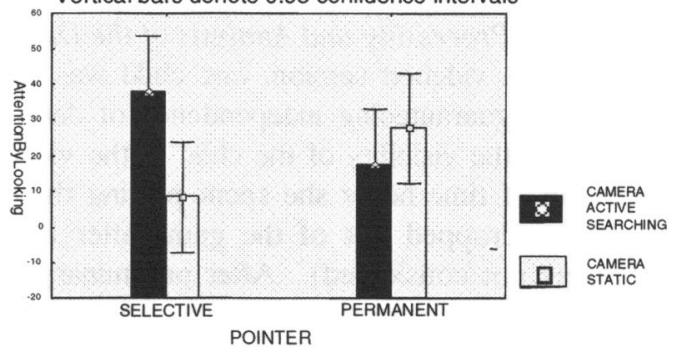

Fig. 5: Analysis of Variance for the variable AttentionBy•Looking under the two states of camera and pointer (active, static) 
Each of the three "AttentionBy•X" variables was subjected to a two-factor (State of Camera, State of Pointer) ANOVA with replication. The results show the significant effect of the interaction between State of Camera and State of Pointer (Fig. 5) for AttentionBy-Looking. Specifically, subjects paid more attention by looking at the robot when either the pointer was selective and camera was active searching or both were static. Children looked relatively less at the robot when camera and pointing were "conflicting" one static and other not. For AttentionBy•Pointing and AttentionBy•Talking no significant affects due to the camera or pointer state were found.

In order to examine in more detail the possible differences in attention children paid to the robot, other children and "else", within each experimental condition, a series of Friedman tests was performed. The Friedman test is a non-parametric two-way analysis of variance (without replication) for rank-ordered data. The test was applied to compare for each subject how often he/she displayed attention towards the three targets (robot, children, else = unknown + other persons). Friedman tests were carried out separately for each of the three attention variables ("Looking at", "Talking to", and "Pointing at") for each of the four experimental conditions (Table 2).

TAB LE 2.

RESULTS OF THE FRIEDMAN TESTS;

FOR EACH VARIABLE DIFFERENCES BETWEEN TARGET WERE TESTED FOR SIGNIFICANCE

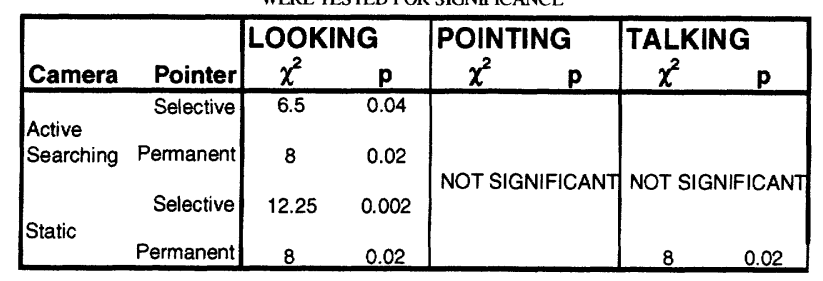

Pointing - No significant differences could be found in the frequency with which the children spent pointing towards the robot, other children or "else" within any of the experimental conditions. Pointing towards the other persons, a teacher or the researcher running the experiment did not occur at all.

Looking - For all experimental conditions we found significant differences in the frequency of "Looking at" the robot, other children or "else". In all cases children looked most often at the robot, less at other children and the least at other persons plus unknown targets.

Talking - Significant differences in how often children talked to the robot, other children or other persons/unknown targets were found only when the camera was static and the pointer was static: in that case children talked significantly more to other children than to the robot or "else".

Although not significant, the same tendency was observed in the experimental condition 3 (camera active searching, pointer permanent). Therefore, these two conditions were further analysed together. The outcome of a Friedman test performed for experimental conditions 1 and 3 together was significant $\left(\chi^{2}=12.074, \mathrm{df}=2, \mathrm{p}=0.002\right)$ and suggests that when the robot's pointer was permanent, irrespective of the camera status, children talked significantly more to other children than to the robot or to other persons/unknown targets.

\section{The Effect of Proximity to the Robot}

Similar analyses as described above were carried out taking the distance to the robot (i.e. the zones in which the subject was positioned in) into account. We did this by entering a code for zone (from 1 to 5 , corresponding with closer to further distance) as an extra factor in the analysis of variance. However, this inclusion lead to serious violations of the assumptions underlying ANOVA (heteroscedasticity = significant differences between the variances) and we therefore had to perform separate tests for each of the four experimental conditions. None of the tests showed significant effects.

\section{CONCLUSIONS AND DISCUSSION}

Embodied non-verbal interactions, such as approach, touch, and avoidance, are fundamental to regulating humanhuman social interactions [Hall, 1968], and these insights have provided guidelines for more recent research, studies and investigations into human reactions to robots, e.g. [Goetz and Kiesler 2002], [Kanda et al., 2003], [Woods, et al, 2004]. Our results however show that even when very simple and general activities are studied, certain human-robot interactions are far from straightforward.

The analysis showed that for all experimental conditions, irrespective of the state of the camera or the pointer status, the highest scored attention variable was looking at the robot. The lack of significant differences in the frequency of pointing at the robot, other children or "else" might be explained by the rare occurrence of this behaviour during the experiment. Pointing at other persons, (except for at other children) did not occur at all, probably as a consequence of obeying social norms (especially in the U.K. this is regarded as rude and the "other person" in the experiment was always an adult. Because all children in our experiment were born and raised in the U.K., we do not think that cultural differences among the children had a strong impact on the results). Alternatively, the "other persons" were not seen as involved in the game.

It is worth mentioning, that some children started mimicking the start 'thumbs up' signal, given to the robot by an experimenter at the beginning of each game. This could be interpreted as trying to influence robot's behaviour or to make a contact with it.

The seemingly "attentive" behaviour of the robot influenced the behaviour of the children in various ways, although not all of the effects are easy to interpret. Overall activity was highest when the hand was permanently pointing, which may be due to boredom, leading in turn to increased talking and interactions with the other children. The heightened attention by looking at the robot showed a surprising result: children were less interested when the camera and pointer were in conflicting states. Possibly this "confused" the children or was counter to their expectations of a "balanced" behaviour on the part of the robot. Apparently, human-robot interactions cannot be understood as one-dimensional responses to simple stimuli.

The only significant differences between talking to the robot, other children or other person/unknown targets were 
found for experimental condition 1 and for the combined data of condition 1 and 3 (permanent pointing). In both cases children more frequently talked to other children and less to the robot or "else". Since in condition 1 the camera and pointer were both static, it is possible that the children interpreted this as a lack of interest from the side of the robot. This in turn might have made the children less interested in the robot and therefore led them to be engaged in a conversation among themselves instead. This is in line with our observation that the overall activity (with regard to the attentional behaviour), of the children was highest when the hand was permanently pointing. This fact and the outcomes of the Friedman test on the combined data from condition 1 and 3 (permanent pointing, independent of the state of the camera) suggest indeed that the robot's selective pointing was a stronger stimulus for the children than the camera. A possible explanation is that its movement was more conspicuous than that of the camera. Alternatively, the fact that the pointer's shape included the form of a human hand might have directed the attention.

No effect of distance to the robot could yet be demonstrated, but at the time of writing only overall attentive behaviour was analysed. We are currently testing the influence of proximity for the separate directed attention variables.

\section{ACKNOWLEDGMENTS}

Thanks go to Christina Kaouri, Iain Werry, Ben Robins, and Aris Alissandrakis for help in carrying out the trails, to Dorota Jagielska for data recording and help in the statistical analysis, and to Alexander Klyubin for his work on the ANNOTATOR Software and other technical assistance.

\section{REFERENCES}

ActivMedia (2005). Activmedia website with details of PeopleBots ${ }^{\mathrm{TM}}$; www.activmedia.com

Bradshaw, J. M. (1997). An introduction to software agents. In: Software Agents, ed. J. M. Bradshaw. Menlo Park, Calif. AAAI Press.

Breazeal, C.L., (2002). Designing Sociable Robots. Massachusetts: The MIT Press, 2002.

COGNIRON, (2004). COGNIRON Project website www.cogniron.org, 2004.

Dautenhahn, K. (1998). The art of designing socially intelligent agents: Science, fiction, and the human in the loop. Applied Artificial Intelligence 12(7-8): 573-617

Dautenhahn, K. and C. L. Nehaniv, Living with Socially Intelligent Agents In: K. Dautenhahn (ed.), Human Cognition and Social Agent Technology, John Benjamins Publishing Company, pp. 415-426.

Dautenhahn, K., B. Ogden, T. Quick (2002). From Embodied to Socially Embedded Agents - Implications for Interaction-Aware Robots, Cognitive Systems Research 3(3): 397-428

Dautenhahn, K., I. Werry (2002) A Quantitative Technique for Analysing Robot-Human Interactions. Proc. IROS2002, Lausanne, 2002 IEEE/RSJ International Conference on Intelligent Robots and Systems, pp. 1132 1138, IEEE Press.

Dautenhahn, K. (2004). Robots We like to Live With?! - A Developmental Perspective on a Personalized Life-Long Robot Companion Proc. IEEE Ro-Man 2004, 13th Annual Workshop on Robot and Human Interactive communication. September 20-22, Kurashiki, Okayama, Japan. IEEE Press, pp. 17-22.
Dryer, D. C. (1999). "Getting personal with computers: How to design personalities for agents.", Applied Artificial Intelligence, 13,:273-295, 1999

Erickson, T. (1997). Designing agents as if people mattered. In: Software Agents, ed. J. M. Bradshaw. Menlo Park, Calif. AAAl Press.

Fong, T., I. Nourbakhsh, and K. Dautenhahn, (2003). A survey of socially interactive robots, Robotics and Autonomous Systems, 42: 143-166, 2003.

Goetz, J. and S. Kiesler, (2002). "Cooperation with a Robotic Assistant" presented at CHI'02 Conference on Human Factors in Computing Systems, New York, USA, 2002.

Hall, E. T. (1966). The Hidden Dimension: Man's Use of Space in Public and Private. The Bodley Head Ltd, London, UK. 1966

Hall, E. T. (1968). Proxemics. Current Anthropology 9(2-3): 83-108. 1968

Kanda, T., Ishiguro, H., Imai, M., T. Ono (2003). Body Movement Analysis of Human-Robot Interaction, Proc. International Joint Conference on Artificial Intelligence (IJCAI 2003), pp.177-182, 2003

Kita, S. (2003). Pointing: a foundational building block in human communication. In S. Kita (Ed.), Pointing: where language, culture, and cognition meet (pp.1-8). Mahwah, NJ: Lawrence Erlbaum.

Kita, S. (2003). Interplay of gaze, hand, torso orientation and language in pointing. In S. Kita (Ed.), Pointing: where language, culture, and cognition meet (pp.307-328). Mahwah, NJ: Lawrence Erlbaum.

Norman D. A., (1994). How might people interact with agents, Communications of the ACM, 37: 68-71.

Norman, D. A. (1997): How people might interact with agents? In: Software Agents, ed. J. M. Bradshaw. Menlo Park, Calif. AAAI Press.

Robins B., K. Dautenhahn, J. Dubowski, (2004). Investigating Autistic Children's Attitudes Towards Strangers with the Theatrical Robot - A New Experimental Paradigm in Human-Robot Interaction Studies, Proc. IEEE Ro-man 2004, IEEE Press, pp. 557-562.

Takeuchi et al. (1994). Simulated facial displays toward social interactions. Proceedings of $\mathrm{CHI} 94$, ACM Press.

VICTEC, (2003). VICTEC Project website www.victec.org, 2003

Woods S., K Dautenhahn, J Schulz, (2004). The Design Space of Robots: Investigating Children's Views. RO-MAN Conference, 13th IEEE Intl. Workshop on Robot and Human Interaction and Communication. Kurashiki, Japan. Sept 2004. 